\title{
Synthesis of single phase Tl-2223 superconductors: How much thallium do we really need?
}

\author{
G M PHATAK, R M IYER, K GANGADHARAN, \\ R M KADAM, P V P S SASTRY, M D SASTRY and \\ J V YAKHMI \\ Chemical Group, Bhabha Atomic Research Centre, Trombay, Bombay 400085, India
}

\begin{abstract}
This paper describes the synthesis and microwave absorption studies of single phase Tl-2223 compound, with $T_{\mathrm{c}}=(129 \pm 1) \mathrm{K}$. The single phase compound has been synthesized using a precursor matrix method. It has been found that Tl-deficient starting composition gives the best results.
\end{abstract}

Keywords. High temperature superconductor; Tl-2223 phase; microwave absorption of superconductors.

\section{Introduction}

The high temperature superconductivity with $T_{c}(R=0)$ up to $125 \mathrm{~K}$ has been established for the homologous series $\mathrm{Tl}_{2} \mathrm{Ca}_{n-1} \mathrm{Ba}_{2} \mathrm{Cu}_{n} \mathrm{O}_{2 n+4}$ (Ginley et al 1988; Gopalakrishnan et al 1988; Hazen et al 1988; Zheng and Hermann 1988; Kikuchi et al 1989; Parkin et al 1988a, b; Torardi et al 1988) with the underlying superconducting phase $\mathrm{Tl}_{2} \mathrm{Ca}_{2} \mathrm{Ba}_{2} \mathrm{Cu}_{3} \mathrm{O}_{10}$ designated as $\mathrm{Tl}-2223$ phase. In these compounds the superconducting phases $\mathrm{Tl}_{2} \mathrm{CaBa}_{2} \mathrm{Cu}_{2} \mathrm{O}_{8}$ (with $T_{c}=108 \mathrm{~K}$ designated as $\mathrm{Tl}-2122$ ) and Tl-2223 phases have been studied extensively and their structural analysis suggests $\sim 10-15 \%$ vacancies at $\mathrm{Tl}$ sites (Hewat et al 1988; Cox et al 1988; Otto et al 1988; Sequeira et al 1988; Syono et al 1989; Zetterer et al 1988). It has been argued by us in an earlier communication (Sequeira et al 1988) that the Tl double layers cannot possibly sustain full $\mathrm{Tl}$ occupancy due to excess positive charge and in the case of Tl-2122 we could in fact successfully substitute $\mathrm{K}^{+}$ions at $\sim 50 \%$ of the $\mathrm{Tl}$-sites and synthesize $\mathrm{Tl}_{1} \mathrm{~K}_{1} \mathrm{Ca}_{1} \mathrm{Ba}_{2} \mathrm{Cu}_{2} \mathrm{O}_{x}$ without any significant deterioration in the superconducting behaviour (Iyer et al 1988; Sequeira et al 1988). This raises doubts whether all the stoichiometric amount of thallium i.e. two thallium atoms per formula are really needed to stabilize the Tl double layers in Tl-2122 and Tl-2223 phases. Eibschutz et al (1988) reported a superconducting phase with zero resistance temperature of $121 \mathrm{~K}$ from a starting composition $\mathrm{Tl}_{0.5} \mathrm{Ca}_{2} \mathrm{Ba}_{2} \mathrm{Cu}_{3} \mathrm{O}_{x}$. The underlying superconducting phase in this compound should be none other than Tl-2223 since the single layer Tl1223 phase is known to have $T_{c}(R=0)$ not exceeding $110 \mathrm{~K}$ (Haldar et al 1988; Parkin et al 1988a).

In this paper, we report the synthesis of single-phase superconducting compounds in $\mathrm{Tl}-\mathrm{Ca}-\mathrm{Ba}-\mathrm{Cu}-\mathrm{O}$ system from a number of different nominal compositions mostly Tl-deficient and have identified the phase to be $\mathrm{Tl}-2223$ and arrived at the optimum $(\mathrm{Tl}+\mathrm{Ca})$ composition which yields the single-phase 2223 compound.

\section{Synthesis of the Tl-based superconductors}

The conventional solid state reaction route namely mixing and heating of the constituent oxides $\mathrm{Tl}_{2} \mathrm{O}_{3}, \mathrm{CaO}, \mathrm{BaO}$ and $\mathrm{CuO}$ has not been found to be effective due to 
the high volatility and low melting point of $\mathrm{Tl}_{2} \mathrm{O}_{3}$. At the reaction temperatures at which the compound is expected to be formed, $\mathrm{Tl}_{2} \mathrm{O}_{3}$ was found to be lost even before the reaction was complete. To avoid this, the Tl-2223 phase has been synthesized in this work using a precursor matrix method-with the precursor of composition $\mathrm{Ca}_{q} \mathrm{Ba}_{2} \mathrm{Cu}_{n} \mathrm{O}_{x}(2<q<3.0$ and $n=2,3)$ heating with $\mathrm{Tl}_{2} \mathrm{O}_{3}$ at high temperature for a short time.

The required matrix has been synthesized by heating a mixture of $\mathrm{BaCO}_{3}, \mathrm{CaCO}_{3}$ and $\mathrm{CuO}$ in the required proportions at $700^{\circ} \mathrm{C}$ for $20 \mathrm{hr}$, followed by heating at $850^{\circ} \mathrm{C}$ for $48 \mathrm{hr}$ and the final heating at $930^{\circ} \mathrm{C}$ for $120 \mathrm{hr}$ under flow of oxygen, with several intermittent grindings and pelletization. Subsequently $\mathrm{Tl}_{2} \mathrm{O}_{3}$ and the matrix $\mathrm{Ca}_{q} \mathrm{Ba}_{2} \mathrm{Cu}_{3} \mathrm{O}_{x}$ have been thoroughly mixed in the required proportions to give a nominal composition $\mathrm{Tl}_{p} \mathrm{Ca}_{q} \mathrm{Ba}_{2} \mathrm{Cu}_{3} \mathrm{O}_{x}$, pelletized into discs with $12 \mathrm{~mm}$ dia $(1 \mathrm{~g})$ and wrapped in nickel foil to reduce thallium losses during heat treatment. The pellets were introduced in a preheated furnace at $900-960^{\circ} \mathrm{C}$ for varying times $(20-40 \mathrm{~min})$ followed by quenching to room temperature by withdrawing from the furnace. The quenched pellet was annealed in oxygen by reintroducing it in a preheated furnace $\left(900-960^{\circ} \mathrm{C}\right)$ and furnace-cooled under oxygen by putting off the furnace. The surface of the pellets in many cases showed higher room temperature resistance which was reduced to milliohms on scrapping the surface. This may be attributed to the contamination with nickel foil. The formation of the compound has been found to be associated with simultaneous increase in volume of the pellet. The preparation conditions are summarized in table 1. The flow diagram for the synthesis is shown schematically in figure 1.

The X-ray diffraction patterns were recorded for all the samples with $\mathrm{Ni}$-filtered $\mathrm{Cu}$ $\mathrm{K} \alpha$ radiation on a Philips PW 1050 wide angle goniometer. For the samples reported in tables 1 and 2 the lines characteristic of the respective precursor matrix were found to be completely absent thus confirming its complete reaction with $\mathrm{Tl}_{2} \mathrm{O}_{3}$. Typical X-ray diffraction patterns for matrix $\mathrm{Ca}_{2} \mathrm{Ba}_{2} \mathrm{Cu}_{3} \mathrm{O}_{x}$ and $\mathrm{Tl}_{1.25} \mathrm{Ca}_{2} \mathrm{Ba}_{2} \mathrm{Cu}_{3} \mathrm{O}_{x}$ are given in figures $2 \mathrm{~A}$ and $2 \mathrm{~B}$.

\section{EPR and electrical resistivity measurements}

We have shown earlier (Sastry et al 1988) that EPR-spectrometric measurements can be effectively used to detect the superconducting phase, even in powder samples, by following the temperature dependance of microwave reflectivity/absorption from a resonant cavity loaded with the superconducting sample. This technique has been extensively used in this study as a first-level evaluation of the quality of superconductors synthesized from different compositions. A Varian V 4502 X-band ESR spectrometer equipped with a closed cycle helium refrigerator has been used in the temperature range $20 \mathrm{~K}$ to $300 \mathrm{~K}$. Electrical resistivity measurements have been carried out between $77 \mathrm{~K}$ and $300 \mathrm{~K}$ using a DC four-probe method with a measuring current of $1 \mathrm{~mA}$. It has been demonstrated in our laboratory earlier that the microwave absorption shows a sharp drop at a temperature where the electrical resistance of cuprate superconductors becomes zero.

Chemical analysis conducted on these samples confirmed that the stoichiometry of the product was close to the starting compositions and the Tl-content was not altered to any measurable extent during synthesis. 


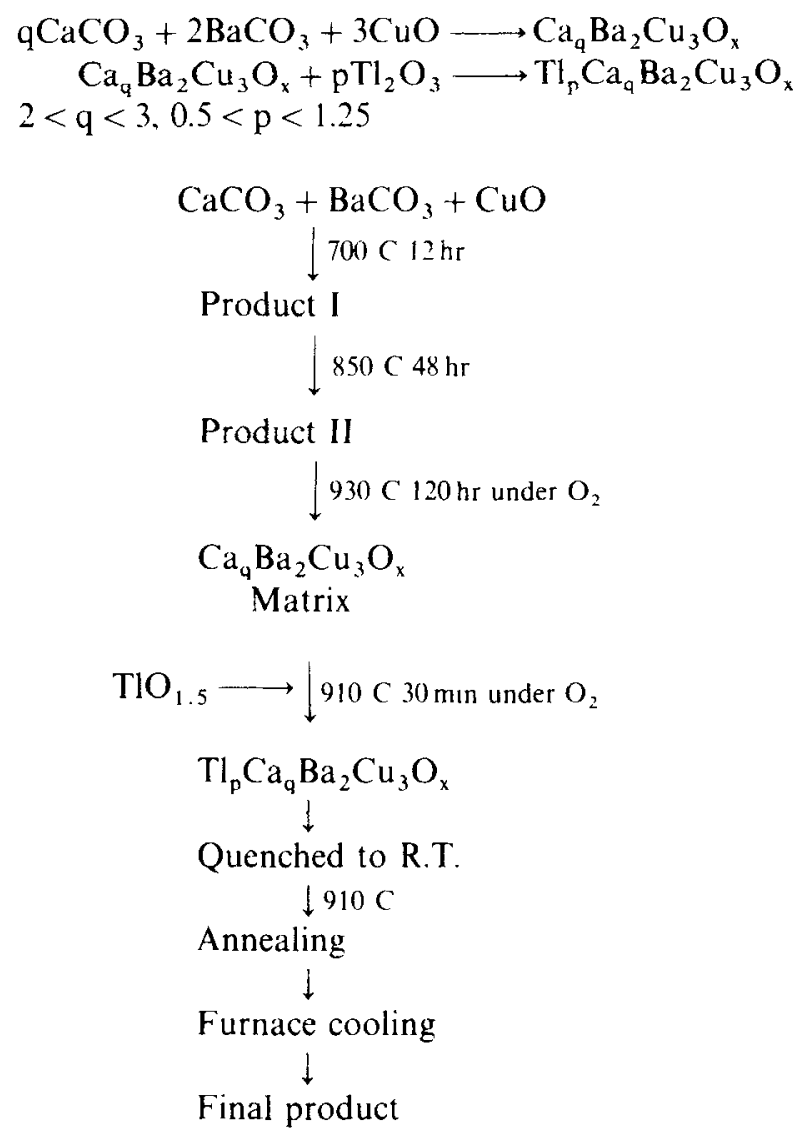

Figure 1. Synthesis of T1-2223 single phase compound matrix reaction method.

Table 1. Preparation conditions and synthesized products from $\mathrm{Tl}_{1} \mathrm{Ca}_{2} \mathrm{Ba}_{2} \mathrm{Cu}_{3} \mathrm{O}_{x}$ as starting nominal composition: Effect of temperature and heating time duration.

\begin{tabular}{|c|c|c|c|}
\hline $\begin{array}{c}\text { Temperature } \\
\text { (C) }\end{array}$ & $\begin{array}{l}\text { Heating time } \\
\text { (min) }\end{array}$ & Products & $T_{c}(K=0)( \pm 1 \mathrm{~K})$ \\
\hline 900 & 30 & $2122+2223$ & Mixed phase compound \\
\hline 905 & 30 & $\begin{array}{l}2223(2122 \\
\text { impurity) }\end{array}$ & 123 \\
\hline \multirow[t]{3}{*}{910} & 20 & 2223 & 123 \\
\hline & 30 & 2223 & 129 \\
\hline & 40 & 2223 & 127 \\
\hline 915 & 30 & 2223 & 127 \\
\hline 920 & 30 & $\begin{array}{l}2223(2122 \\
\text { impurity) }\end{array}$ & 125 \\
\hline 930 & 30 & $\begin{array}{l}\text { Compound melts } \\
\text { with partial } \\
\text { decomposition }\end{array}$ & 115 \\
\hline 940 & 30 & $\begin{array}{l}\text { Compound melts } \\
\text { with decomposition }\end{array}$ & \\
\hline 960 & 20 & -do- & \\
\hline
\end{tabular}


Table 2. Synthesis of Tl-2223 from Tl-deficient nominal compositions: Effect of calcium and thallium concentration on $T_{c}(R=0)$

\begin{tabular}{lccc}
\hline Nominal composition & Phase formed & $T_{c}(R=0)$ & $(\mathrm{Tl}+\mathrm{Ca})$ \\
\hline $\mathrm{Tl}_{0.5} \mathrm{Ca}_{3} \mathrm{Ba}_{2} \mathrm{Cu}_{3} \mathrm{O}_{x}$ & 2223 & 130 & 3.50 \\
$\mathrm{TlCa}_{2} \mathrm{Ba}_{2} \mathrm{Cu}_{3} \mathrm{O}_{x}$ & 2223 & 130 & 3.00 \\
$\mathrm{Tl}_{1.25} \mathrm{Ca}_{2} \mathrm{Ba}_{2} \mathrm{Cu}_{3} \mathrm{O}_{x}$ & 2223 & 121 & 3.25 \\
$\mathrm{Tl}_{3} \mathrm{Ca}_{2} \mathrm{Ba}_{2} \mathrm{Cu}_{3} \mathrm{O}_{x}$ & 2223 & 115 & 5.00 \\
$\mathrm{TlCa}_{2.25} \mathrm{Ba}_{2} \mathrm{Cu}_{3} \mathrm{O}_{x}$ & 2122 & & \\
$\mathrm{TlCa}_{2.5} \mathrm{Ba}_{2} \mathrm{Cu}_{3} \mathrm{O}_{x}$ & 2223 & 123 & 3.25 \\
$\mathrm{TlCa}_{3} \mathrm{Ba}_{2} \mathrm{Cu}_{3} \mathrm{O}_{x}$ & 2223 & 120 & 3.50 \\
$\mathrm{Tl}_{1.25} \mathrm{Ca}_{2.25} \mathrm{Ba}_{2} \mathrm{Cu}_{3} \mathrm{O}_{x}$ & 2223 & 118 & 4.00 \\
\hline
\end{tabular}

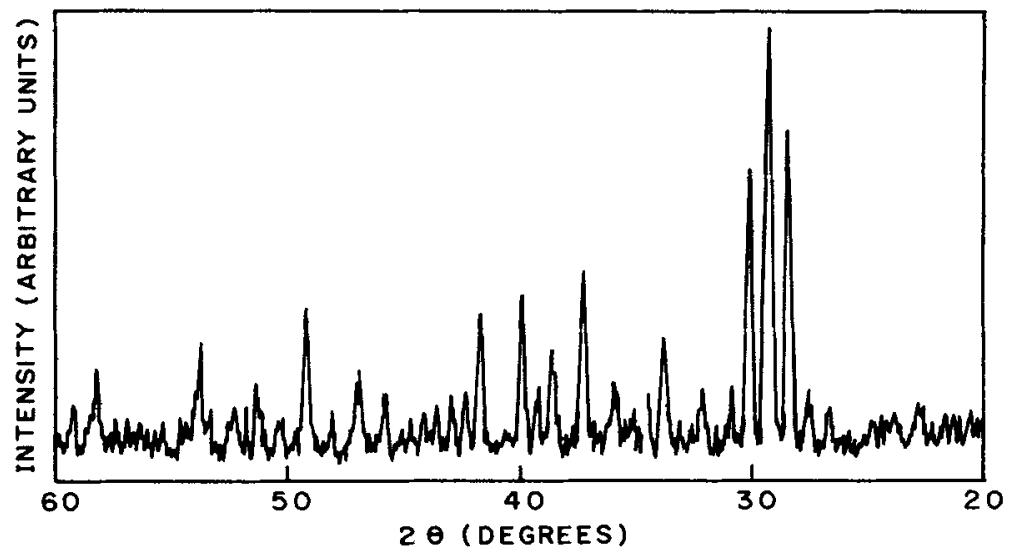

Figure 2A. X-ray diffraction pattern of precursor matrix $\mathrm{Ca}_{2} \mathrm{Ba}_{2} \mathrm{Cu}_{3} \mathrm{O}_{x}$.

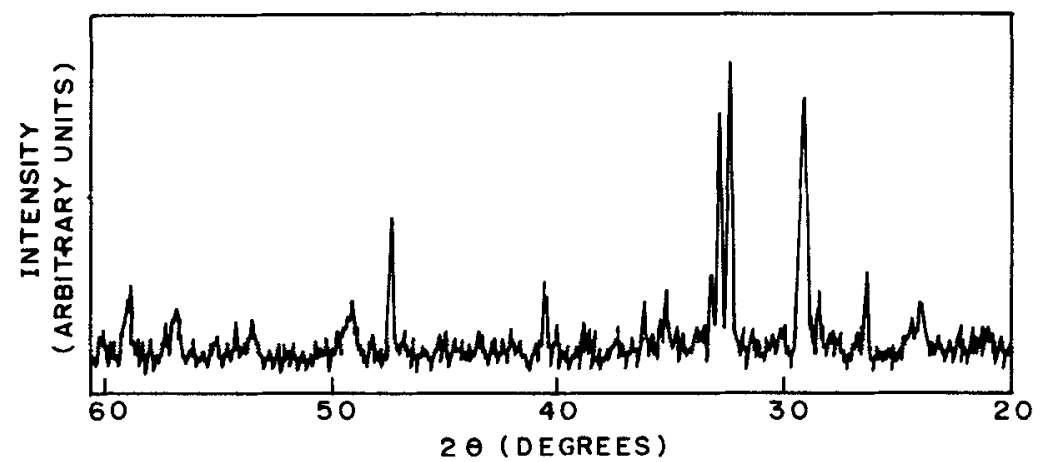

Figure 2B. X-ray difraction pattern of $\mathrm{Tl}_{1.25} \mathrm{Ca}_{2} \mathrm{Ba}_{2} \mathrm{Cu}_{3} \mathrm{O}_{x}$. The absence of precursor matrix lines may be noted. 


\section{Results and discussion}

The results of the phase analysis using $X$-ray powder diffraction technique and the superconducting transition temperatures from microwave and electrical resistivity measurements for a number of compounds synthesized under different and identical heat treatment protocol as described above from a variety of starting compositions are described in tables 1 and 2, respectively. The microwave data on the compositions listed in table 2 confirmed the presence of only one superconducting transition in each case for samples 1 to 8 except for sample 4 which exhibited two transitions. Figure 3 depicts a typical behaviour of microwave reflectivity data as a function of temperature for samples 3 and 4 . This clearly shows that the sample with Tl-rich nominal composition $\mathrm{Tl}_{3} \mathrm{Ca}_{2} \mathrm{Ba}_{2} \mathrm{Cu}_{3} \mathrm{O}_{x}$ shows two superconducting transitions whereas the Tl-deficient sample with nominal composition of $\mathrm{Tl}_{1.25} \mathrm{Ca}_{2} \mathrm{Ba}_{2} \mathrm{Cu}_{3} \mathrm{O}_{x}$ shows a metallic behaviour in the normal state and exhibits a sharp transition at $121 \mathrm{~K}$, with no evidence of any other superconducting phase down to $20 \mathrm{~K}$. An examination of the data shown in tables 1 and 2 brings out the following important aspects of the present results:

(i) The synthesis of Tl-2223 from identical Tl-deficient nominal starting compositions is strongly affected by the reaction temperature and the temperature zone for the formation of stable Tl-2223 phase is very narrow viz $910-915^{\circ} \mathrm{C}$.

(ii) As low as $25 \%$ of the stoichiometric requirement of Tl-content (viz. sample 1 in table 2) is adequate to sustain Tl-2223 structure in single phase.

(iii) For wide composition range with the $\mathrm{Tl}$ :Ca ratio varying from $0.5: 3,1: 2,1.25: 2$, $3: 2,1: 2.25,1: 2.5,1: 3,1.25: 2.25$, (samples 1 to 8 except 4 in table 2 ) the 2223 structure results with $T_{c}(R=0)$ value ranging from $130 \mathrm{~K}$ to $116 \mathrm{~K}$, combined stoichiometry of $(\mathrm{Tl}+\mathrm{Ca})$ between 3 and 4 invariably leads to the formation of $\mathrm{Tl}-2223$ phase alone. (iv) At a constant $\mathrm{Tl}$ concentration in the starting material, with increase in calcium concentration, $T_{c}(R=0)$ value decreases from $130 \mathrm{~K}$ to $118 \mathrm{~K}$ (table 2 , samples $2,5-7$ ) and from $121 \mathrm{~K}$ to $116 \mathrm{~K}$ (table 2, samples 3 and 8 ).

(v) At a constant calcium concentration in the starting material, increase in $\mathrm{Tl}$ concentration results in the decrease in $T_{c}(R=0)$ in 2223 phase and further increase in

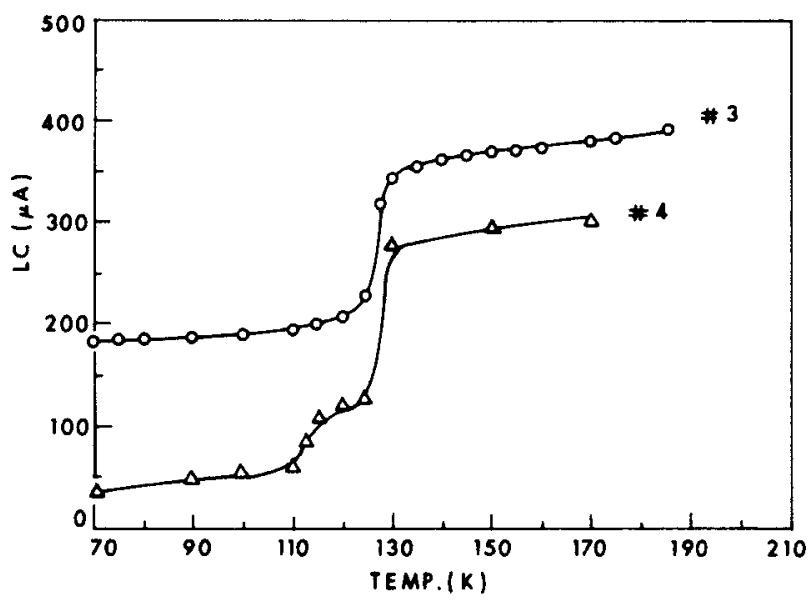

Figure 3. Temperature dependence of microwave absorption (measured as change in leakage currents LC) for samples 3 and 4. 
$\mathrm{Tl}$ concentration to $\mathrm{Tl}_{3}$ results in the formation of mixed phase compound with emergence of $T 12122$ phase in the product in significant concentration (table 2 , samples 2 to 4$)$.

(vi) The formation of Tl-2223 from nominal thallium-deficient starting compositions e.g. $\mathrm{Tl}_{1} \mathrm{Ca}_{2} \mathrm{Ba}_{2} \mathrm{Cu}_{3} \mathrm{O}_{x}$ is an interesting finding of this investigation. This is probably due to the existence of vacancies at calcium sites which play a crucial role in the formation of pure $\mathrm{Tl}-2223$ phase, the thallium deficiency aiding migration of Ca ions to Tl sites (Iyer et al 1989).

\section{Conclusions}

It is established that using Tl-deficient starting nominal compositions enables one to prepare pure Tl-2223 phase with a $T_{c}(R=0) 129 \pm 1 \mathrm{~K}$. Increase in thallium concentration $>\mathrm{Tl}_{1.25}$ results in the formation of $\mathrm{Tl}-2122$ as impurity phase. Compositions containing $(\mathrm{Tl}+\mathrm{Ca})>4$ lead to the stabilization of both $\mathrm{Tl}-2223$ and Tl-2122 phases.

\section{References}

Cox D E, Torardi C C, Subramanian M A, Gopalakrishnan J and Sleight A W 1988 Phys. Rev. B38 6624 Eibschutz M, Vanwitert L G, Grader G S, Gryogy E N, Glarum S J, Grodkiewicz W H, Kyle T R, White A E, Short K T and Zydzik G J 1988 Appl. Phys. Lett. 53911

Ginley D S, Venturini E L, Kwak J F, Baughman R J, Carr M J, Hlava F F, Schirber J E and Morosin B 1988 Physica C152 217

Gopalakrishnan I K, Sastry P V P S S, Gangadharan K, Phatak G M, Yakhmi J V and Iyer R M 1988 Appl. Phys. Lett. 53414

Haldar P, Chen K, Maheswaran B, Roig-Janicki, Jaggi N K, Markiewicz R S and Griessen B C 1988 Science 2411198

Hazen R M, Finger L W, Angel R J, Prewitt C T, Ross N L, Hadidiacos C G, Heaney P J, Veblen D R, Shen Z Z, Ali El and Hermann A M 1988 Phys. Rev. Lett. 601657

Hewat A W, Hewat E A, Brynstad J, Mook H A and Specht E D 1988 Physica C152 438

Iyer R M, Sastry P V P S S, Phatak G M, Gopalakrishnan I K, Gangadharan K and Sastry M D 1988 Physica C152 505

Iyer R M, Phatak G M, Gangadharan K, Sastry M D, Kadam R M, Sastry P V P S S and Yakhmi J V 1989 Physica C150 155

Kikuchi M, Kajitani T, Suzuki T, Nakajima S, Hiraja K, Kobayashi N, Iwasaki $H$, Syono Y and Muto $Y$ 1989 Jpn. J. Appl. Phys. 28 L382

Otto H H, Zetterer T and Renk K F 1988 Naturwissenschaften 75509

Parkin S S P, Lee V Y, Nazzal A I, Savoy R, Huang T C, Gorman G and Beyers R 1988a Phys. Rev. B38 6531

Parkin S S P, Lee V Y, Engler E M, Nazzal A I. Huang T C, Gorman G, Savoy R and Beyers R 1988b Phys. Rev. B38 2539

Sastry M D, Kadam R M, Babu Y, Dalvi A G I, Gopalakrishnan I K, Sastry P V P S S and Iyer R M 1988 Physica C153-155 1667

Sequeira A, Rajagopal H, Gopalakrishnan I K, Sastry P V P S S, Phatak G M, Yakhmi J V and Iyer R M 1988 Physica C156 599

Sheng Z Z and Hermann A M 1988 Nature (London) 33255

Syono Y, Kikuchi M, Nakajima S, Suzuki T, Oku T, Hiraga K, Kobayashi H, Iwasaki H and Muto Y 1989 Symposium on High Temperature Superconductivity, MRS Spring Mtg., San Diego, USA

Torardi C C. Subramanian M A. Calabrese J C, Gopalakrishnan J. Morrisey K J, Askew T R, Flippen R B, Chowdhry U and Sleight A W 1988 Science 240631

Zetterer T, Otto H H, Lugert G and Renk K F 1988 Z. Phys. B73 321 